\title{
Solidification Behavior in the Presence of External Fields: Part I
}

\author{
LANG YUAN (10 ${ }^{1,3}$ and ANDREW $\mathrm{KAO}^{2,4}$ \\ 1.-College of Engineering and Computing, University of South Carolina, Columbia, SC 29201, USA. \\ 2.-School of Computing and Mathematical Sciences, University of Greenwich, London SE10 \\ 9LS, UK. 3.—e-mail: langyuan@cec.sc.edu. 4.—e-mail: A.Kao@greenwich.ac.uk
}

The introduction of external fields, such as electromagnetic fields, ultrasonic excitation, and mechanical shearing, to solidification processes has encompassed novel applications to refine grains, homogenize segregation, break up agglomeration of particles, and prevent defect formation. Advances in experimental methods, materials characterization, and computational modeling have led to new insights into controlling the microstructure and defects during solidification. This two-part topic, "Solidification Behavior in The Presence of External Fields," highlights the most recent investigations into the use of external fields in a broad range of industrial manufacturing processes.

The first part focuses on the use of electromagnetic fields, a key technique that is typically used to modify fluid flow within a melt. This phenomenon, known as magnetohydrodynamics (MHD), has many uses in solidification processing, such as materials refining and production of alloys. Liquid metal MHD is governed by the Lorentz force, which is introduced by the external magnetic field. Applying a time-varying magnetic field introduces electromagnetic stirring that redistributes the solute. Conversely, for static magnetic fields, the force can act as a brake to fluid flow through the electromagnetic damping effect, whereas when electrical currents are present in the system, a static magnetic field can also be a driver for fluid flow, for example, through the interaction with inherent thermoelectric currents. In this part, four papers cover the different perspectives of the above phenomenon and provide excellent examples of applications of electromagnetic fields in solidification processes.

Lang Yuan and Andrew Kao are guest editors for the Solidification Committee of TMS and coordinated the topic Solidification Behavior in the Presence of External Fields in this issue.

(Received August 7, 2020; accepted August 7, 2020; published online August 26, 2020)
Continuous casting is the most widely used process to manufacture steel. Electromagnetic fields play an important role in removing defects in recent process developments. The first article, by Seongmook Cho and Brian G. Thomas, is titled "Electromagnetic Effects on Solidification Defect Formation in Continuous Steel Casting." It briefly reviews typical solidification defects, including subsurface hooks, particle capture, deep oscillation marks, depressions, cracks, breakouts, segregation, and shrinkage, and their formation mechanisms, in continuous casting of steel. It then focuses on the effects of electromagnetic fields on the solidification phenomena and their influences on the formation of solidification defects. Based on the discussion, practical strategies are provided for the reduction of defects and improvement of steel quality.

By applying an industrial strand electromagnetic stirring in continuous casting, the second article, titled "Effect of electromagnetic stirring on the solidification behavior of high-magnetic induction grain-oriented silicon steel continuous casting slab" by $\mathrm{Li}$ et al., studies the intensity of a string of electromagnetic fields on grain morphology, central porosity, and carbon segregation for the silicon steel. With increasing current intensities, the equiaxed zone is increased with increasing carbon segregation. Meanwhile, the central porosity is gradually improved by increasing the current intensity, but it cannot be eliminated. The observations suggest that electromagnetic fields may not be beneficial to grain-oriented silicon steel for better magnetic properties.

Beyond the application of electromagnetic fields in continuous casting, the third article by $\mathrm{He}$ et al. studies its impacts on $\mathrm{Al}-\mathrm{Mg}$-Si alloy in the twin-roll casting process. The article is titled "Improvement of spatial solute elements inhomogeneity and mechanical properties of twin-roll cast Al-Mg-Si alloy in the presence of electromagnetic fields." It is found that the eutectic phase was decreased by either a static magnetic field or a pulse electric field. When both fields are applied simultaneously, the 
eutectic phase shows the lowest volume fraction, and the distribution of alloying elements in the thickness direction of the twin roll cast sheet is the most uniform with minimal segregation. The homogenized microstructure contributes to improved mechanical properties of the $\mathrm{Al}$ alloy.

Examining the physical phenomena at the microscale under a static magnetic field, the last article in this first part by Kao et al., titled "Magnetic effects on the microstructure and solute plume dynamics of a directionally solidifying GaIn alloy," presents a comprehensive fundamental study to reveal how thermoelectric magnetohydrodynamics (TEMHD) affect segregation within the dendritic structures by in situ $\mathrm{x}$-ray imaging and multiphysics microstructure modeling. Both experiments and simulations show that TEMHD can lead to the formation of a stable segregated 'chimney' channel by increasing solutal buoyancy in the flow direction. Moreover, the resulting pressure difference across the solidification front introduces a secondary hydrodynamic phenomenon that subsequently causes solute plume migration. This study sheds light on mitigating segregation channels in directional solidification.

The papers in this special topic, Solidification Behavior in The Presence of External Fields, can be accessed via the October issue's Table of Contents page at https://link.springer.com/journal/11837/72/ 10/page/1.

\section{CONFLICT OF INTEREST}

On behalf of all authors, the corresponding authors state that there is no conflict of interest.

Publisher's Note Springer Nature remains neutral with regard to jurisdictional claims in published maps and institutional affiliations. 